\title{
Reciprocal insulation analysis of Hi-C data shows that TADs represent a functionally but not structurally privileged scale in the hierarchical folding of chromosomes
}

\author{
Yinxiu Zhan, ${ }^{1,2}$ Luca Mariani, ${ }^{3,9}$ Iros Barozzi, ${ }^{4}$ Edda G. Schulz, ${ }^{3,10}$ Nils Blüthgen, ${ }^{5,6}$ \\ Michael Stadler, ${ }^{1,7}$ Guido Tiana, ${ }^{8}$ and Luca Giorgetti ${ }^{1}$ \\ ${ }^{1}$ Friedrich Miescher Institute for Biomedical Research, Basel, CH-4058, Switzerland; ${ }^{2}$ University of Basel, $\mathrm{CH}-4003$ Basel, Switzerland; \\ ${ }^{3}$ Institut Curie, PSL Research University, CNRS UMR3215, INSERM U934, 75248 Paris Cedex 05, France; ${ }^{4}$ Genomics Division, \\ Lawrence Berkeley National Laboratory, Berkeley, California 94720, USA; Institute of Pathology, Charité-Universitätsmedizin Berlin, \\ 10117 Berlin, Germany; ${ }^{6}$ Interdisciplinary Research Institute for the Life Sciences, Humboldt University, 10115 Berlin, Germany; \\ ${ }^{7}$ Swiss Institute of Bioinformatics, CH-4058 Basel, Switzerland; ${ }^{8}$ Department of Physics and Center for Complexity and Biosystems, \\ University of Milano and Istituto Nazionale di Fisica Nucleare, 20133, Milano, Italy
}

\begin{abstract}
Understanding how regulatory sequences interact in the context of chromosomal architecture is a central challenge in biology. Chromosome conformation capture revealed that mammalian chromosomes possess a rich hierarchy of structural layers, from multi-megabase compartments to sub-megabase topologically associating domains (TADs) and sub-TAD contact domains. TADs appear to act as regulatory microenvironments by constraining and segregating regulatory interactions across discrete chromosomal regions. However, it is unclear whether other (or all) folding layers share similar properties, or rather TADs constitute a privileged folding scale with maximal impact on the organization of regulatory interactions. Here, we present a novel algorithm named $\mathrm{CaTCH}$ that identifies hierarchical trees of chromosomal domains in Hi-C maps, stratified through their reciprocal physical insulation, which is a single and biologically relevant parameter. By applying $\mathrm{CaTCH}$ to published $\mathrm{Hi}-\mathrm{C}$ data sets, we show that previously reported folding layers appear at different insulation levels. We demonstrate that although no structurally privileged folding level exists, TADs emerge as a functionally privileged scale defined by maximal boundary enrichment in CTCF and maximal cell-type conservation. By measuring transcriptional output in embryonic stem cells and neural precursor cells, we show that the likelihood that genes in a domain are coregulated during differentiation is also maximized at the scale of TADs. Finally, we observe that regulatory sequences occur at genomic locations corresponding to optimized mutual interactions at the same scale. Our analysis suggests that the architectural functionality of TADs arises from the interplay between their ability to partition interactions and the specific genomic position of regulatory sequences.
\end{abstract}

[Supplemental material is available for this article.]

Characterizing the three-dimensional organization of chromosomes in mammalian cells is a central challenge, especially in light of determining how regulatory sequences such as enhancers and promoters interact and ensure precise control of gene expression during development. Methods based on chromosome conformation capture (3C) and notably 4C, 5C, and Hi-C, which measure physical interaction frequencies of genomic loci in the three-dimensional nuclear space, have revealed that mammalian chromosomes possess a rich hierarchy of structural layers (Gibcus and Dekker 2013). Each chromosome is partitioned in multi-megabase ' $\mathrm{A}$ ' and ' $\mathrm{B}$ ' compartments, reflecting the associations of alternating large regions of active and inactive chromatin (Lieberman-Aiden et al.2009). Compartments are further subdivided into topological-

Present addresses: ${ }^{9}$ Division of Genetics, Department of Medicine, Brigham and Women's Hospital and Harvard Medical School, Boston, MA 02115, USA; 1 Max-Planck-Institute for Molecular Genetics, Otto-Warburg-Laboratory, Max Planck Research GroupRegulatory Networks in Stem Cells, 14195 Berlin, Germany Corresponding author: luca.giorgetti@fmi.ch

Article published online before print. Article, supplemental material, and publication date are at http://www.genome.org/cgi/doi/10.1101/gr.212803.116. ly associating domains (TADs), contiguous sub-megabase genomic regions within which the chromatin fiber preferentially associates (Dixon et al. 2012; Nora et al. 2012), which are further partitioned into smaller substructures and 'contact domains' (Berlivet et al. 2013; Phillips-Cremins et al. 2013; Rao et al. 2014). Finally, as a further level of complexity, TADs also interact with each other into "meta-TAD" trees that extend up to several Mb (Fraser et al. 2015). Given the cell population-averaged nature of 3C-based experiments, the observed nested hierarchies of interaction domains may arise as statistical patterns resulting from an average over millions of alternative conformations of the chromatin fiber (Fudenberg and Mirny 2012; Giorgetti et al.2014; Junier et al. 2015).

Although more than one mechanism might give rise to TADs and sub-TAD structures, CTCF (CCCTC-binding factor) and the cohesin complex appear to be largely responsible for the establishment and maintenance of TADs and sub-TAD structures and

(C) 2017 Zhan et al. This article is distributed exclusively by Cold Spring Harbor Laboratory Press for the first six months after the full-issue publication date (see http://genome.cshlp.org/site/misc/terms.xhtml). After six months, it is available under a Creative Commons License (Attribution-NonCommercial 4.0 International), as described at http://creativecommons.org/licenses/by-nc/4.0/. 
boundaries. Indeed, CTCF and cohesin are enriched at TAD boundaries (Dixon et al. 2012; Van Bortle et al. 2014), but they also bind pervasively within TADs and are involved in the formation of sub-TAD structure (Rao et al. 2014; de Wit et al. 2015; Sanborn et al. 2015), although the molecular mechanisms that lead to structure formation are unclear (Merkenschlager and Nora 2016). In addition, open chromatin and active transcription positively correlate with the presence of TADs and sub-TAD structure (Hou et al. 2012; Phillips-Cremins et al. 2013; Ulianov et al. 2015), and active histone modifications are enriched at TAD boundaries (Dixon et al. 2012), suggesting that interactions between active regulatory sequences may contribute to establish chromosomal architecture. However, transcription does not seem to be strictly needed for maintaining TAD boundaries (Nora et al. 2012).

Irrespective of the mechanisms underlying their formation, genetic evidence suggests that TADs contribute to establish correct interaction patterns between enhancers and promoters (Symmons et al. 2014; Lupiáñez et al. 2015; Franke et al. 2016). Consistent with this, transcriptional coregulation of neighboring genes is favored within TADs during differentiation (Nora et al. 2012) and upon transcriptional responses to external stimuli (Le Dily et al. 2014). TADs are thought to act, on the one hand, by increasing the chances that regulatory elements meet each other in the three-dimensional space within a single domain, and on the other hand, by segregating physical interactions across boundaries, thus decreasing the probability that deleterious interactions occur. Hence, the degree to which each TAD is insulated with respect to its neighbors may be an important parameter in the establishment of the correct regulatory connections. It is, however, unclear whether the functional attributes that have been observed at the level of TADs (namely the ability to constrain enhancer-promoter interactions and promote transcriptional coregulation) are specific to the folding layer of TADs themselves, and if so, why those properties emerge at this particular folding scale.

A comprehensive analysis that considers all previously identified topological levels simultaneously and compares them to one another in terms of their functional and physical properties is currently lacking. A small number of algorithms that identify hierarchies of topological domains are available (Filippova et al. 2014; Lévy-Leduc et al. 2014; Shin et al. 2015; Weinreb and Raphael 2015; Chen et al. 2016; Shavit et al. 2016). However, none of them provides a quantitative description of how the various layers of domains differ from one another. In addition, these algorithms define hierarchies of interaction domains depending on one or more parameters that do not have a clear biological or structural interpretation. To overcome these limitations, we developed a novel algorithm called CaTCH (Caller of Topological Chromosomal Hierarchies) that identifies nested topologies of structural domains in Hi-C data sets based on a single parameter, the reciprocal physical insulation between domains, which is a simple and biologically relevant measure. Here, we describe the $\mathrm{CaTCH}$ algorithm and report the results of comparing the structural and functional properties of domains across the folding hierarchy of the mouse genome.

\section{Results}

\section{CaTCH: an algorithm to detect and stratify nested hierarchies of topological domains}

In order to comprehensively describe the multiscale organization of chromosomal folding hierarchies, we developed an algorithm that segments Hi-C interaction maps into multiple alternative sets of domains and stratifies them according to a single parameter. We adopted a thermodynamic interpretation of Hi-C data sets (Fudenberg and Mirny 2012) in which the Hi-C signal between a pair of loci is proportional to the probability of detecting them in proximity across the cell population. For any pair of adjacent chromosomal domains $A$ and $B$, we then defined their reciprocal insulation $(R I)$ as

$$
\begin{aligned}
R I(A, B)= & {\left[P_{\text {in }}(A)+P_{\text {in }}(B)-P_{\text {out }}(A, B)\right] /\left[P_{\text {in }}(A)+P_{\text {in }}(B)\right] } \\
& \times 100,
\end{aligned}
$$

where $P_{\text {in }}$ and $P_{\text {out }}$ are the average Hi-C counts within a domain and across two adjacent domains, respectively (Fig. 1A; see Methods section). Small (large) values of $R I$ thus correspond to domains that are poorly (strongly) insulated from their first neighbors. For example, $70 \%$ reciprocal insulation means that the average Hi-C counts across the boundaries of two adjacent domains are $70 \%$ smaller than the average counts within the two domains.

Given a certain degree of reciprocal insulation, the algorithm merges all consecutive domains whose reciprocal insulation is lower than the chosen threshold (Fig. 1B; see Methods section), similarly to what is commonly performed by agglomerative hierarchical clustering (Hastie et al. 2009). Thus, for any reciprocal insulation threshold, detected domains are at least insulated by the threshold value. By smoothly increasing the threshold on the insulation, the algorithm detects a set of domains that are increasingly more insulated, larger, and containing previous domain layers. This results in a nested hierarchy of differentially insulated domains (Fig. 1C). We dubbed this algorithm $\mathrm{CaTCH}$, for Caller of Topological Chromosomal Hierarchies.

A key property of $\mathrm{CaTCH}$ is that it does not rely on the tuning of any free parameter to identify one particular folding scale. The only parameter in the algorithm is the reciprocal insulation threshold itself, which is systematically varied to define and stratify the entire hierarchy of domains, rather than tuned to identify a single domain set. Moreover, unlike parameters in existing approaches to identify multiscale domain structures in Hi-C data sets (Filippova et al. 2014; Lévy-Leduc et al. 2014; Shin et al. 2015; Weinreb and Raphael 2015; Chen et al. 2016; Shavit et al. 2016), the reciprocal insulation is a biologically relevant measure estimating how efficiently a domain is physically insulated from its immediate neighbors. CaTCH is provided as an R package at https://github.com/ zhanyinx/CaTCH_R (source code can be found in Supplemental Methods).

\section{Sub-TAD contact domains, TADs, and compartments emerge at different levels in the folding hierarchy}

We first applied $\mathrm{CaTCH}$ to published Hi-C data sets from female mouse ESCs (Giorgetti et al. 2016) binned at 20-kb resolution. As expected, when increasing the reciprocal insulation parameter, the algorithm detected increasingly larger and fewer topological domains (Fig. 1C), with 5\% changes in reciprocal insulation translating into $\sim 30 \%$ changes in the number and size of domains (Supplemental Fig. S1a). We found a similar trend when analyzing other cell types, notably neural precursor stem cells (NPCs) derived from the same ESC line (Giorgetti et al. 2016) and the mouse B-cell lymphoma CH12 cell line (Supplemental Fig. S1b; Rao et al. 2014). In ESCs, below $40 \%$ reciprocal insulation domains are too small $(<100 \mathrm{~kb}$ on average) to be characterized with data at $20-\mathrm{kb}$ resolution. At higher insulation values, however, we detected domains 
A

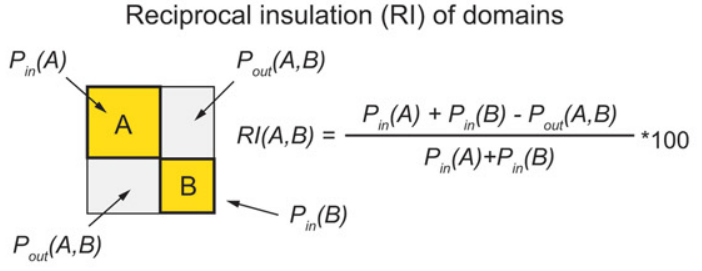

B

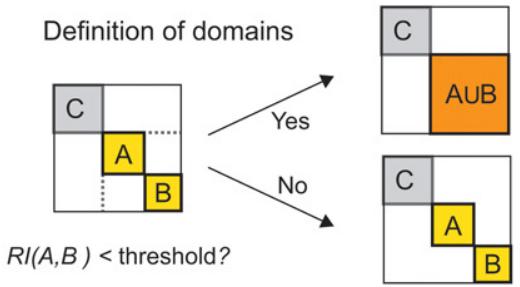

C
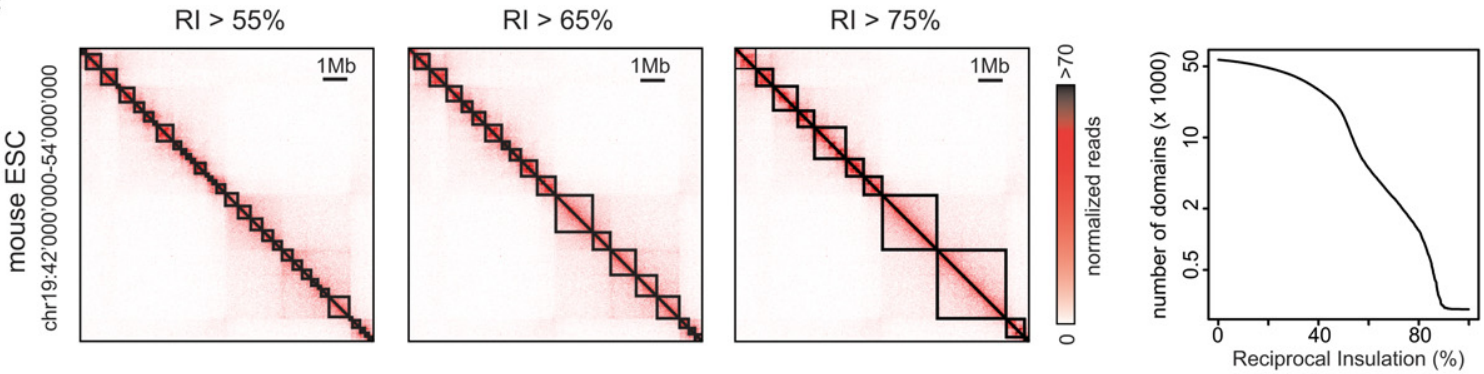

D

$\mathrm{RI}>55 \%$ chr6:87'020'000-89' $120^{\prime} 000$

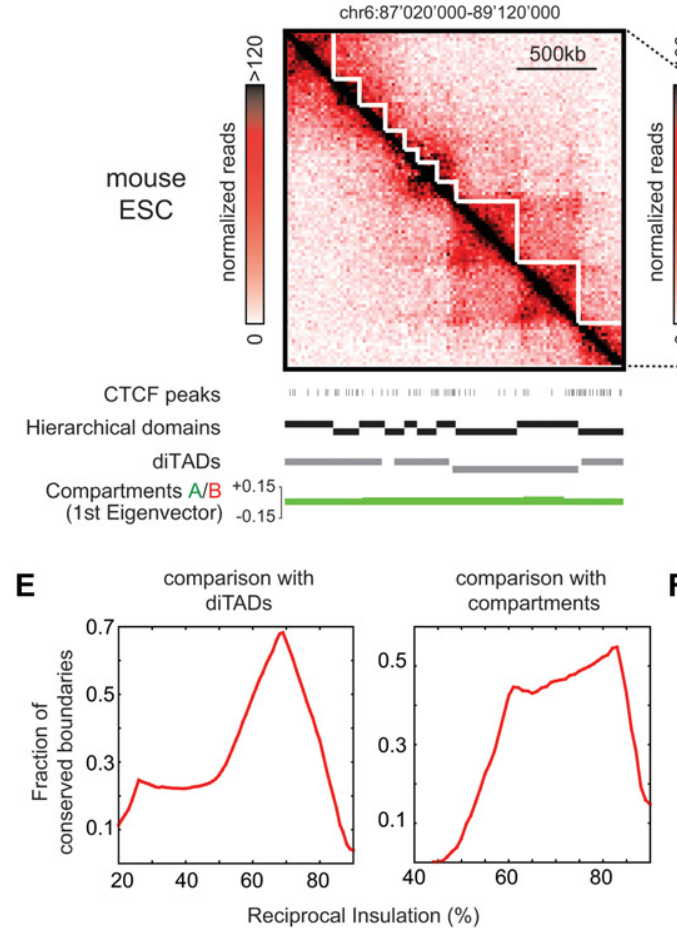

$\mathrm{RI}>69 \%$

chr6:81' 880 '000-89' $120^{\prime} 000$

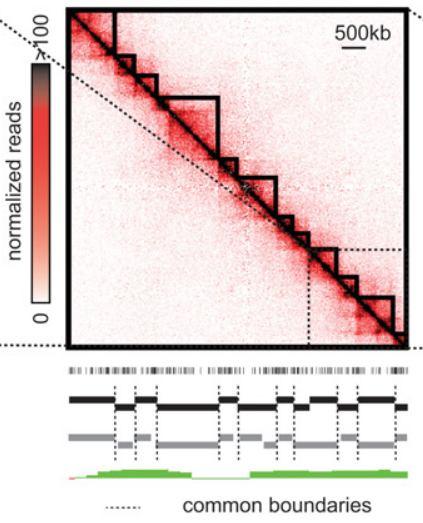

$\mathbf{F}$

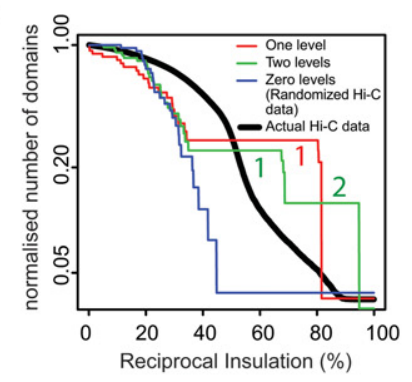

$\mathrm{RI}>85 \%$ chr6:73'240'000-89' 120 '000
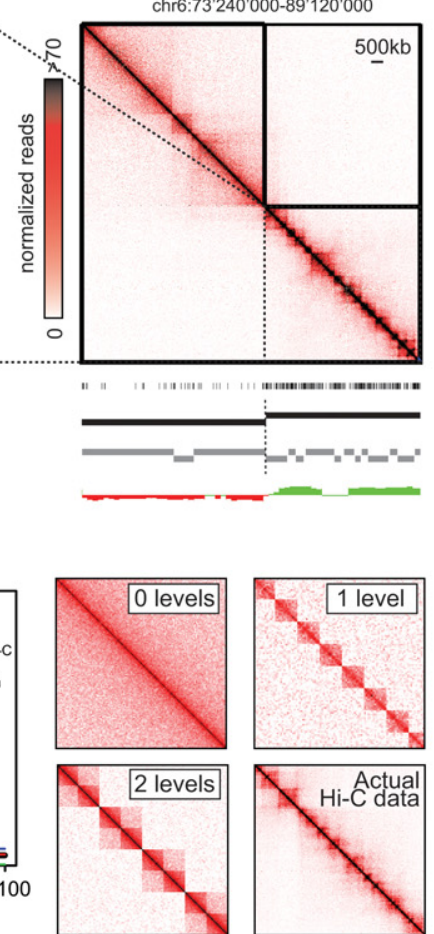

Figure 1. Schematic description of reciprocal insulation and the domain-calling algorithm. (A) Schematic representation of reciprocal insulation (RI) between two fictitious domains $\mathrm{A}$ and $\mathrm{B}$ in $\mathrm{Hi}-\mathrm{C}$ data. $(B)$ The $\mathrm{CaTCH}$ algorithm merges two adjacent domains if their reciprocal insulation is smaller than a given threshold. (C) (Left three panels) Examples of sets of domains defined in mouse ESCs Hi-C data (20-kb binning) imposing different threshold on RI. (Right) Number of domains detected in ESC as a function of RI. (D) Sub-TAD contact domains (left), directionality index-based TADs (middle), and A/B compartments (right) are identified at different RI values. (E) Fraction of boundaries of diTAD (left) and compartments (right) overlapping with boundaries of domains identified by CaTCH as a function of RI. $(F)$ (Left) Number of domains detected by CaTCH as a function of RI in the real genome (black line), or in computationally generated contact maps with zero (blue), one (red), or two preferential folding levels (green). The corresponding heat maps are shown in the four right panels. Numbers of domains were normalized to the initial step ( $0 \%$ insulation) to allow comparison.

with a size (180 kb on average) in the range of sub-TAD structures and 'contact domains' identified in previous studies (Fig. 1D, left; Supplemental Fig. S1c; Berlivet et al. 2013; Phillips-Cremins et al. 2013; Rao et al. 2014). More than $60 \%$ of domain boundaries identified at $55 \%$ reciprocal insulation contain at least a CTCF peak identified in a published ChIP-seq data set (Cheng et al. 2014), consistent with the notion that sub-TAD structures are highly cor- related with CTCF binding (Phillips-Cremins et al. 2013). In addition, although the resolution of the Hi-C data set is not high enough to distinguish most of the CTCF-associated 'loop' signals as in Rao et al. (2014), we noticed that $\sim 45 \%$ of domains at this scale have at least one CTCF peak at both boundaries (Supplemental Fig. S1d). Of the CTCF-delimited domains, however, only 35\% had convergent CTCF sites (compared to 98\% of 'loop 
domains,' defined as contact domains with strong interaction between boundaries in Rao et al. 2014). This is largely due to the fact that the domains identified in the latter study are a subset of domains detected by $\mathrm{CaTCH}$ at 55\% reciprocal insulation (see below); however, a direct comparison between the two domain sets is not possible, due to the lack of ESCs Hi-C data sets in the study by Rao et al. (2014).

To determine the actual overlap between domains identified by $\mathrm{CaTCH}$ and contact domains described in Rao et al. (2014), we analyzed the 10 -kb-resolution $\mathrm{Hi}-\mathrm{C}$ data that were obtained in $\mathrm{CH} 12$ cells in the same study. Maximal overlap between the two domain sets occurred at $62 \%$ reciprocal insulation in $\mathrm{CH} 12$ (Supplemental Fig. S1e), where 78\% of boundaries of previously identified contact domains are also detected by $\mathrm{CaTCH}$. However, CaTCH detects more domains than those identified in Rao et al. (2014) (Supplemental Fig. S1f), which explains the lower proportion of domains delimited by convergent CTCF sites in our data set. Thus, sub-TAD contact domains are detected by CaTCH as relatively lowly insulated regions.

We next sought to identify the scale in the folding hierarchy where domains detected by $\mathrm{CaTCH}$ most closely resemble TADs. Since directionality index analysis (Dixon et al. 2012) has been used to define TAD boundaries in a number of previous studies, here we adopted this benchmark definition of TADs and refer to these domains as 'diTADs' (directionality index TADs). It is important to point out that the set of diTADs identified in a Hi-C experiment depends on the value of two tunable parameters, one setting a limit to the maximal genomic distance over which Hi-C interactions are evaluated (Supplemental Fig. S1g) and the other defining the minimum acceptable size of domains. We set these parameters to $2 \mathrm{Mb}$ and $80 \mathrm{~kb}$, respectively, as used previously (Dixon et al. 2012), to build a reference set of diTADs. This resulted in the identification of 2220 diTADs with a median size of $840 \mathrm{~kb}$, compatible with earlier analyses in mouse ES cells (Dixon et al. 2012). The best overlap between hierarchical domains detected by $\mathrm{CaTCH}$ and diTADs occurred at around 69\% reciprocal insulation (Fig. 1D, center), where $\sim 70 \%$ of diTAD boundaries coincide with hierarchical domain boundaries (Fig. 1E, left) and their size distributions are very similar (Supplemental Fig. S1h). Domains detected by our algorithm at this scale are slightly (although not significantly) smaller than diTADs (median size $760 \mathrm{~kb}$ vs. $840 \mathrm{~kb}$ ) (Supplemental Fig. S1h,i). Most (74\%) CaTCH boundaries not corresponding to TADs indeed divide diTADs in smaller domains (Supplemental Fig. S1j). Thus, diTADs are detected by $\mathrm{CaTCH}$ as domains that are more robustly insulated than contact domains.

At even higher reciprocal insulation, hierarchical domains detected by $\mathrm{CaTCH}$ correspond to regions of increasingly longerrange associations between TADs, in the range of meta-TADs described in Fraser et al. (2015), themselves contained into even larger domains occurring at very high insulation (around 85\%) (Fig. $1 \mathrm{D}$, right). These domains largely overlap with consecutive stretches of genomic sequence belonging to either the 'A' or ' $\mathrm{B}$ ' compartments (Lieberman-Aiden et al. 2009), as detected by eigenvector analysis (Imakaev et al. 2012) on the same ESCs Hi-C data set (Fig. 1D, right, 1E, right). Consistent with the notion that $\mathrm{A} / \mathrm{B}$ compartments represent predominantly active/inactive chromatin, using publicly available ChIP-seq data sets in ESCs (Supplemental Table S1), we found that the difference in histone modification patterns within vs. across domain boundaries is maximized at this scale (Supplemental Fig. S1k).

Thus, CaTCH identifies a continuous spectrum of nested self-interacting chromosome domains, stratified as contiguous genomic regions with differential reciprocal insulation levels. Previously described chromosomal structures such as sub-TAD contact domains, TADs, and groups of TADs emerge at different scales within the nested folding hierarchy and are characterized by increasing reciprocal insulation levels.

\section{A continuous nested hierarchy of topological associating structures}

We then sought to determine whether one or more privileged reciprocal insulation levels exist among the folding hierarchy and correspond to any of the previously reported folding layers. If such level(s) existed, some simple fundamental quantities, such as the number or size of domains detected by the CaTCH algorithm, would have a discontinuous behavior as a function of the reciprocal insulation parameter. To exemplify this concept, we computationally generated simplified control contact maps by artificially imposing the presence of zero, one, or two scales of domains, separated by sharp transitions in contact probabilities between consecutive layers (see Methods section; Fig. 1F). For these controls, CaTCH detected a number of plateaus in the size (or number) of domains equal to the number of distinct hierarchical levels (Fig. 1F, left), irrespective of the genomic size of the domains (Supplemental Fig. S11). Compared to these controls, the ESC genome does not exhibit any structurally privileged scale, at least for domains defined using reciprocal insulation as a measure (black line in Fig. 1F), irrespective of whether the entire genome is considered or select regions that belong to either the A (active) or B (inactive) compartment (Supplemental Fig. S1b). A similar trend can be observed in NPCs and CH12 cells (Supplemental Fig. S1b), suggesting that no obvious privileged structural scale exists in ESCs and differentiated cell types. As a notable consequence, TADs do not appear as a natural intrinsic structural scale in the nested hierarchy of domains. This prompted us to investigate whether functional properties that have been previously attributed to TADs specifically emerge at the TAD scale or are rather widespread among the folding hierarchy.

\section{Enrichment in active histone marks is maximized at the scale of TADs}

TAD boundaries have been shown to be enriched in histone modifications associated with active transcription (Dixon et al. 2012). We therefore analyzed publicly available ChIP-seq data sets in ESCs (Supplemental Table S1) and computed the enrichment for distinct histone marks at the boundaries of the domains across all the scales in the folding hierarchy. Marks associated with active transcription showed a steady increase in enrichment as a function of reciprocal insulation and reached a plateau at the level of diTADs ( 69\%) (Supplemental Fig. S2a). Thus, although active histone marks show widespread enrichment across the folding hierarchy, they are maximally enriched at the scale of TADs and TAD aggregates (meta-TADs and compartments). Consistent with previous results (Dixon et al. 2012), the H3K9me3 repressive mark was found depleted at many levels in the folding hierarchy and notably at the level of diTADs (Supplemental Fig. S2a).

\section{CTCF clustering at boundaries is maximized at the scale of TADs}

Consistent with its putative role in establishing and/or maintaining chromosomal structure, CTCF is enriched at boundaries of contact domains (Berlivet et al. 2013; Phillips-Cremins et al. 2013; Rao et al. 2014), TADs (Dixon et al. 2012), and meta-TAD

\section{Genome Research}

www.genome.org 
trees (Fraser et al. 2015). We therefore computed the enrichment in the number of CTCF ChIP-seq peaks (Cheng et al. 2014) at domain boundaries at all folding scales. As expected, CTCF binding is enriched at boundaries of every level across the folding hierarchy; however, CTCF enrichment is maximized at the scale of TADs, and in particular at $\sim 65 \%$ reciprocal insulation (Fig. $2 \mathrm{~A}$ ) corresponding to domains that are slightly less insulated than diTADs detected using standard directionality index parameters. Identical results were found by using the input-normalized CTCF ChIP-seq signal per boundary, rather than the number of ChIP-seq peaks (Supplemental Fig. S2b). We noticed that maximal CTCF enrichment is due to both a maximal number of boundaries containing at least one CTCF peak and a maximal average number $(\sim 1.9)$ of CTCF peaks per boundary (Supplemental Fig. S2c), which are mostly found within the $40 \mathrm{~kb}$ upstream of or downstream from the boundary (Supplemental Fig. S2d).

Domains at $65 \%$ reciprocal insulation are $20 \%$ smaller compared to 'standard' diTADs (600 kb vs. $840 \mathrm{~kb}$ median size) (Fig. 2B) and frequently originate from the splitting of one diTAD into two or more smaller domains (Supplemental Fig. S2e). The majority ( 70\%) of these 'new' boundaries have at least one occupied CTCF site, which explains the slightly higher enrichment in CTCF compared to standard diTADs. However, by systematically varying the values of parameters in the directionality index algorithm, we identified alternative sets of diTADs where CTCF enrichment is higher than standard diTADs and comparable to (although slightly lower than) 65\% RI domains (Supplemental Fig. S2f).
Importantly, these alternative directionality index domains correspond to domains detected by $\mathrm{CaTCH}$ in the $65 \%-70 \%$ reciprocal insulation range (Supplemental Fig. S2f, arrows). This confirms that the TAD scale is characterized by maximal CTCF enrichment at boundaries compared to other folding levels. We will hereafter refer to domains identified by $\mathrm{CaTCH}$ at $65 \%$ minimal reciprocal insulation simply as TADs, since they constitute the set of domains with maximal CTCF enrichment.

Reciprocal orientation of CTCF binding sites has been shown to be highly predictive of strong long-range 'looping' interactions (Rao et al. 2014; de Wit et al. 2015; Guo et al. 2015; Vietri Rudan et al. 2015). We therefore assessed the orientation of the two most internal CTCF motifs on either side of each domain and found that, at the scale of TADs, the fraction of domains where CTCF motifs were convergent was maximal (Supplemental Fig. S2g, left), with $\sim 22 \%$ of domains possessing convergent binding sites. Thus, both CTCF clustering and head-to-head orientation of the most internal CTCF motifs are maximized at the scale of TADs. Using available CTCF ChIP-Seq data sets (Phillips-Cremins et al. 2013; Cheng et al. 2014), we found that, in both NPCs and CH12 cells, CTCF enrichment at boundaries showed a similar trend as in ESCs, with a peak around 58\% and $82 \%$ reciprocal insulation in NPCs and CH12, respectively (Fig. 2C). The fraction of domains with convergent CTCF motifs peaked at the same $R I$ values (Supplemental Fig. S2g). Importantly, despite the difference in absolute reciprocal insulation values, the number and size of domains at maximal CTCF enrichment were extremely

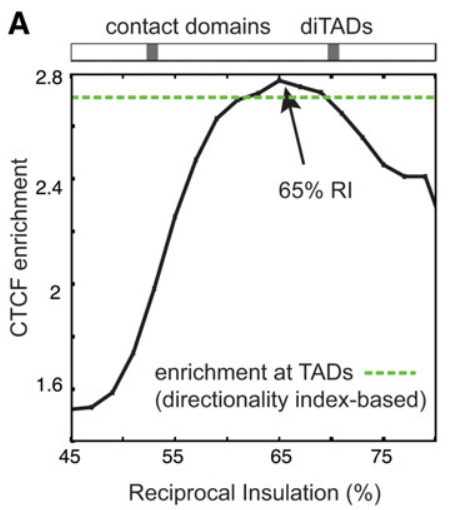

B
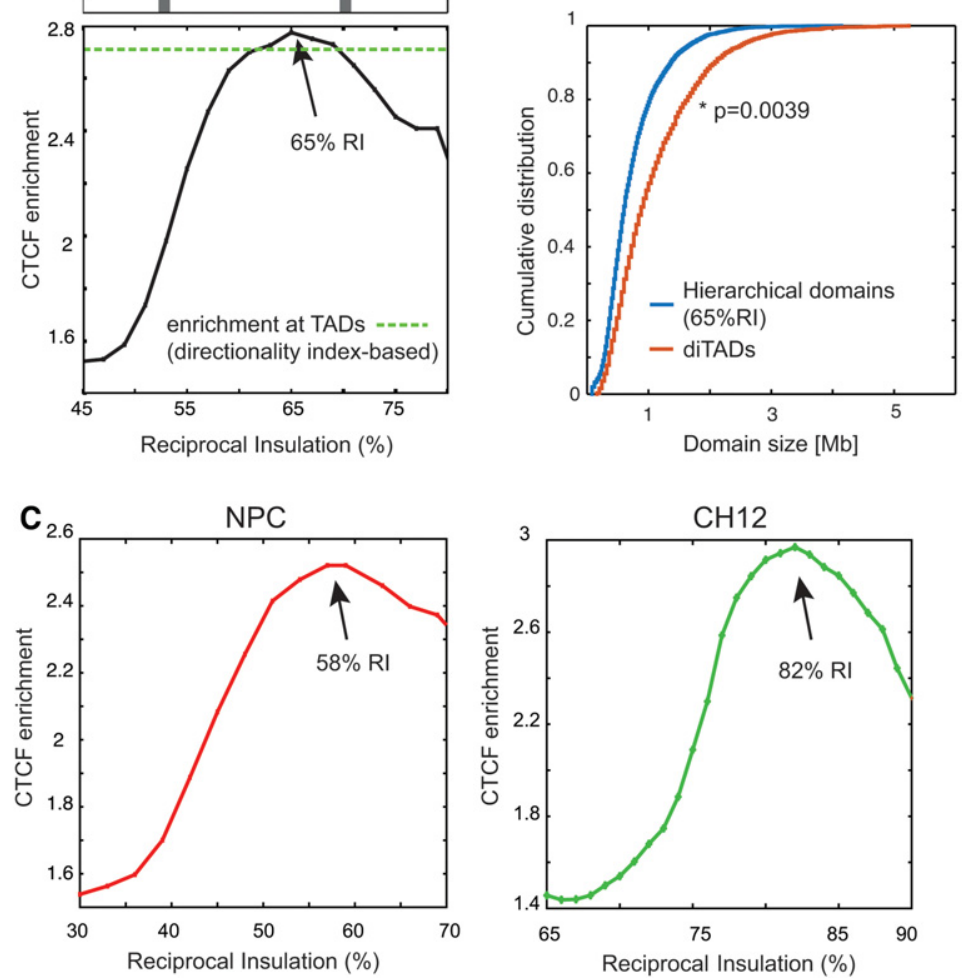

D
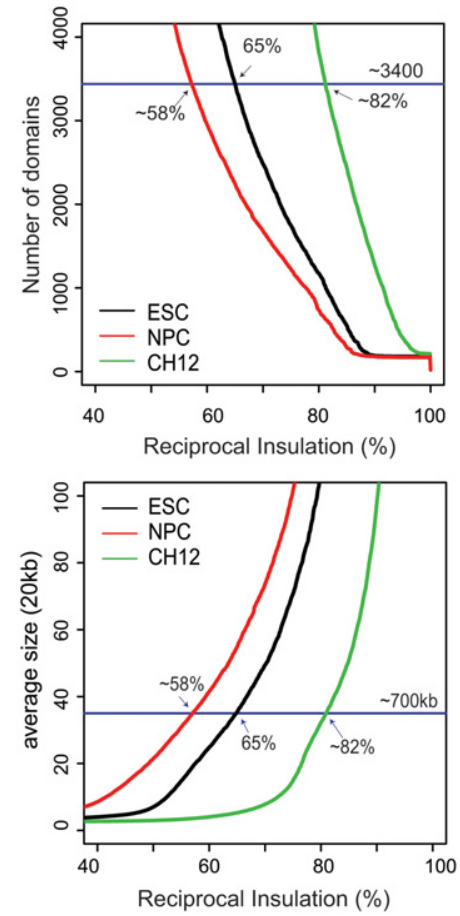

Figure 2. CTCF clustering at domain boundaries is maximal at the scale of TADs. (A) CTCF enrichment at domain boundaries is widespread among the folding hierarchy in mouse ES cells. However, maximal enrichment occurs at $65 \% \mathrm{RI}$, where it is slightly higher compared to TADs identified by directionality index analysis (diTADs). (B) Domains at $65 \%$ are slightly smaller than diTADs identified on the same data set. (C) CTCF enrichment at domain boundaries in NPCs and $\mathrm{CH} 12$ cells shows a similar trend as in ESCs, with maxima located at $58 \%$ and $82 \% \mathrm{RI}$ in NPCs and CH12, respectively. (D) The number and size of domains defined by maximal CTCF enrichment at boundaries are similar in ESCs, NPCs, and $\mathrm{CH} 12$ cells. 
similar across the three cell types (Fig. 2D). In addition, conservation of boundaries across the three cell types was also found to be maximal at the same scale, with $\sim 70 \%$ of boundaries conserved between any two cell types (Supplemental Fig. S2h). Thus, the scale of TADs appears in the entire folding hierarchy not only as the domain scale that maximizes CTCF enrichment at boundaries, but also as the scale where domains are most conserved across cell types.

We next sought to determine any confounding effect on the determination of the optimal $R I$ value due to experimental factors, such as different sequencing depth of Hi-C libraries or different versions of the Hi-C protocol. To study the effect of sequencing coverage, we performed $\mathrm{CaTCH}$ and CTCF enrichment analysis on a down-sampled ESC Hi-C data set obtained by reducing by half the total number of sequenced reads. CTCF enrichment at domain boundaries was maximized at very similar reciprocal insulation value as in the full data set (67\% vs. 65\%) (Supplemental Fig. S2i), largely corresponding to the same set of domains (Supplemental Fig. S2k). Thus, sequencing depth is not likely to have a strong impact on the reciprocal insulation values where TADs appear. Next, to understand the impact of using Hi-C data sets obtained using different experimental protocols, we performed a comparative analysis of two data sets obtained in mouse fetal liver cells (Nagano et al. 2015) using either the 'dilution' (LiebermanAiden et al. 2009; Belton et al. 2012) or the 'in situ' ligation protocols (Nagano et al. 2013; Rao et al. 2014). Using a published CTCF data set (Cheng et al. 2014), we found that maximal CTCF enrichment occurred at different reciprocal insulation values (Supplemental Fig. S2j,k), with the dilution protocol leading to smaller values compared to the in situ protocol (70\% vs. $77 \%$ ). This is consistent with the lower insulation values where TADs appear in NPC and ESC (where Hi-C was performed with the dilution protocol [Giorgetti et al. 2016]) compared to $\mathrm{CH} 12$ cells (in situ protocol) and is compatible with the previous observation that the in situ protocol leads to sharper TAD boundaries (Nagano et al. 2015). These results point at $\mathrm{Hi}-\mathrm{C}$ protocol variants as a main determinant of reciprocal insulation and suggest that the scale of TADs occurs in the $58 \%-70 \%$ range $(64 \% \pm 6 \%)$ in dilution Hi-C data sets and in the $77 \%-82 \%$ range $(80 \% \pm 3 \%)$ in the in situ experiments that were analyzed.

\section{Transcriptional coregulation during differentiation is maximal at the scale of TADs}

Motivated by the finding that CTCF and active histone marks enrichment at boundaries is maximal at the scale of TADs, we set out to determine whether domains at this scale encompass maximally coregulated genes, which is a further important functional attribute proposed for TADs (Nora et al. 2012; Le Dily et al. 2014). For this, we performed strand-specific RNA-seq on total RNA from the ESC and NPC lines in which the Hi-C had been performed (Giorgetti et al. 2016). Strand specificity allowed us to unambiguously assign up- or down-regulated transcripts in the case of two overlapping transcriptional units. For all levels in the folding hierarchy, we then set out to determine how many domains are transcriptionally coregulated during the differentiation from ESCs to NPCs.

We defined a domain to be down- (up-) coregulated at the empirical $P \leq 0.05$ level if the number of down- (up-) coregulated genes in the domain is larger than in $95 \%$ of cyclically permutated genomes (see Methods section). For each insulation level and the corresponding domain set, we then calculated a $Z$-score as the dif- ference between the number of coregulated domains that were observed in the real genome and the mean number of coregulated domains detected in 2000 randomizations of the genome (Fig. 3A; see Supplemental Methods), weighted by its standard deviation. Interestingly, at all insulation levels, the subset of domains that we detected to be up- or down-regulated at the level of $P \leq$ 0.05 show maximal transcriptional changes during development (Fig. 3B; Supplemental Fig. S3a). Thus, domains with a high level of transcriptional coregulation largely overlap with those where the most dramatic changes in gene expression occur during differentiation.

At the level of TADs in ESC (65\% insulation), we detected 114 coregulated domains, accounting for $\sim 4 \%$ of the total number of TADs and $\sim 10 \%$ of those exhibiting expression changes during differentiation ( $\geq 2$ up- or down-regulated genes). This represents a $>2.5$-fold enrichment relative to the values expected in randomized genomes. Moreover, the number of coregulated TADs (65\% reciprocal insulation) is very similar to that observed at the level of TADs in the context of the acute transcriptional response to progesterone in a human breast cancer cell line (Le Dily et al. 2014).

For genes that are down-regulated during differentiation, the $Z$-score is maximum at the scale of TADs (Fig. 3C). To check the robustness of the analysis against stochastic fluctuation of expression changes, we studied the behavior of $Z$-scores upon randomly reshuffling ( $n=1000) 10 \%$ of genes. For $66 \%$ of these partially reshuffled genomes, the maximum $Z$-score was found to be located within a $4 \%$ interval around $65 \%$ reciprocal insulation $(63 \%-66 \%)$ (Supplemental Fig. S3b), supporting the robustness of the result. This analysis suggests that TADs in ESCs constitute a functionally privileged scale, maximizing the coregulation of genes that are down-regulated during the differentiation into neural precursor stem cells.

The behavior of up-regulated genes was remarkably different, with low (if any) enrichment in transcriptional coregulation within domains below $75 \%$ reciprocal insulation (Fig. 3D) and maximal enrichment at the scale of $\mathrm{A} / \mathrm{B}$ compartments (>80\%). We reasoned that this could be due to the fact that not all TADs identified in ESCs are predictive for transcriptional coregulation of genes that become activated during differentiation. We thus performed the same analysis on domains identified in NPCs and found that coregulation of both down- and up-regulated genes is maximized within domains defined in NPCs around 58\% reciprocal insulation (Fig. 3E,F). This is the set of TADs defined in NPCs by maximal CTCF clustering at their boundaries (see Fig. 2C). We verified that these results are not affected by the presence of an inactive $\mathrm{X}$ chromosome in NPCs, as maximal coregulation was observed at the scale of TADs even when expression changes of X-linked genes (excluding genes that escape $\mathrm{X}$ inactivation in this clone [Giorgetti et al. 2016]) were corrected to account for their monoallelic expression in NPCs (Supplemental Fig. S3c; Supplemental Methods).

Thus, TADs defined in the initial developmental stage (ESCs) are the scale where transcriptional coregulation of down-regulated genes is maximal, whereas the set of domains that better favors the coregulation of up-regulated genes corresponds to TADs defined in the final state (NPCs). This can be largely explained by the fact that, although most TAD boundaries $(\sim 70 \%)$ are conserved between ESCs and NPCs, a significant fraction ( $30 \%)$ is not. In particular, although most up-regulated TADs are conserved and detected in both ESCs and NPCs, we detected 20\% more up-regulated TADs in NPCs than in ESCs, corresponding to domains that

\section{Genome Research}

www.genome.org 
A

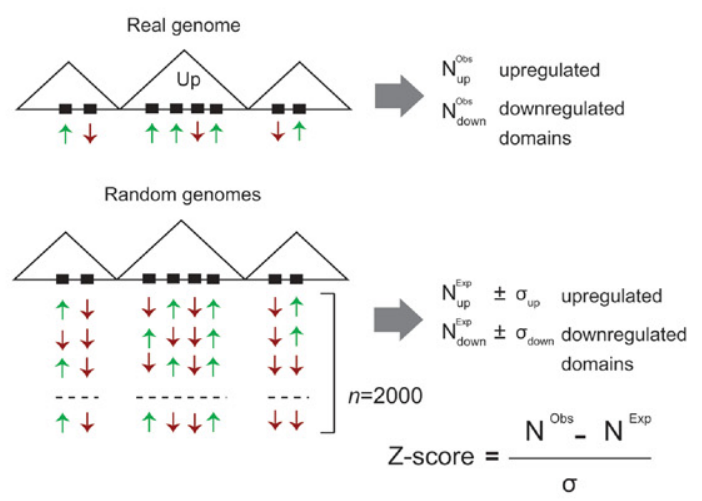

B

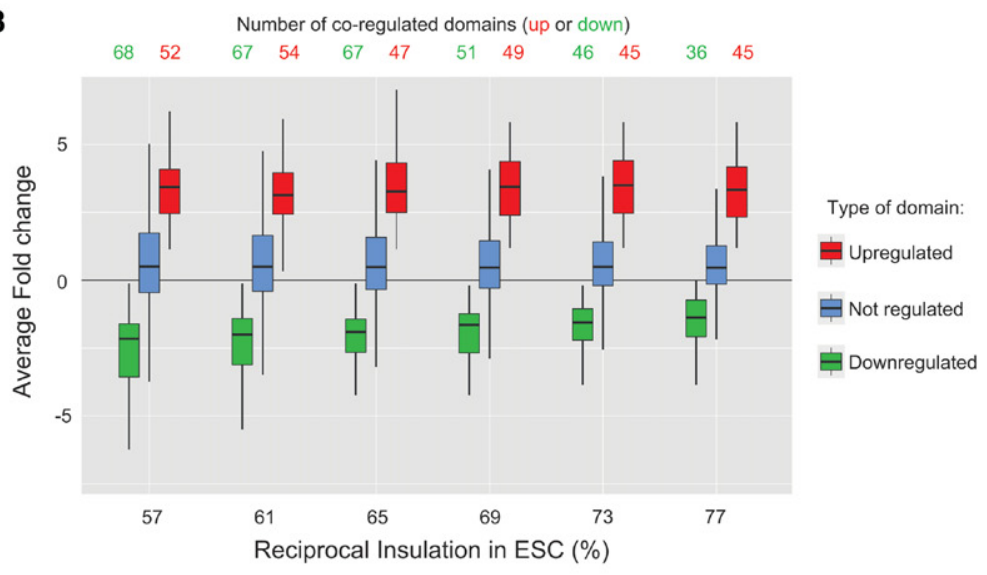

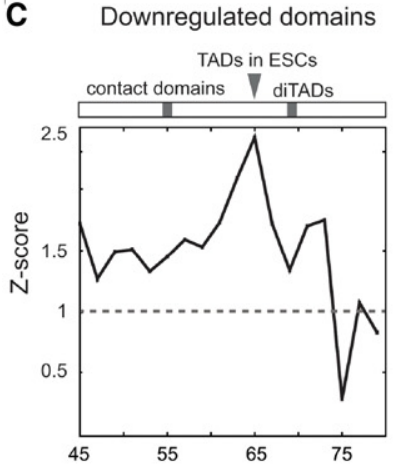

Reciprocal Insulation in ESC (\%)
D Upregulated domains

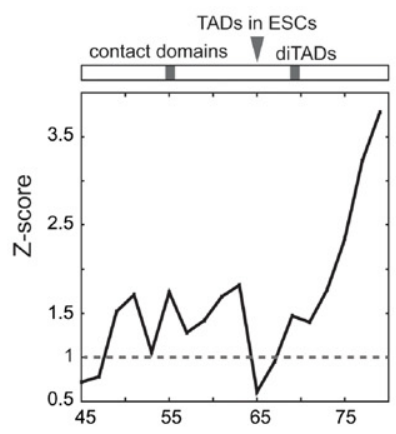

Reciprocal Insulation in ESC (\%)

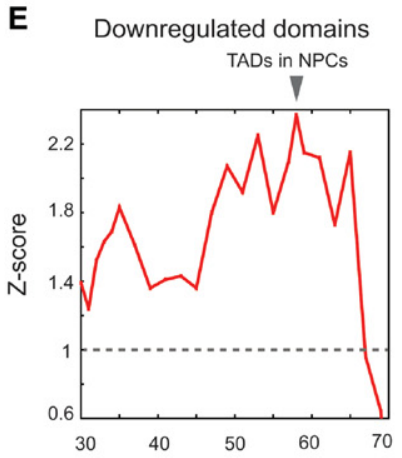

Reciprocal Insulation in NPC (\%)

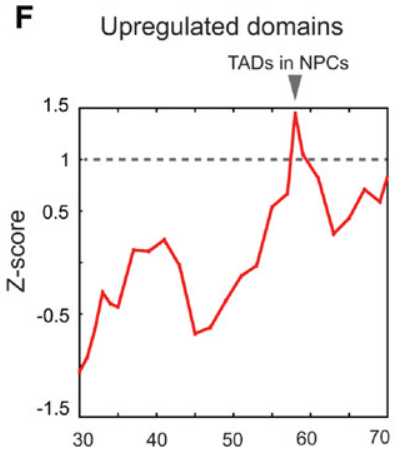

Reciprocal Insulation in NPC (\%)
G
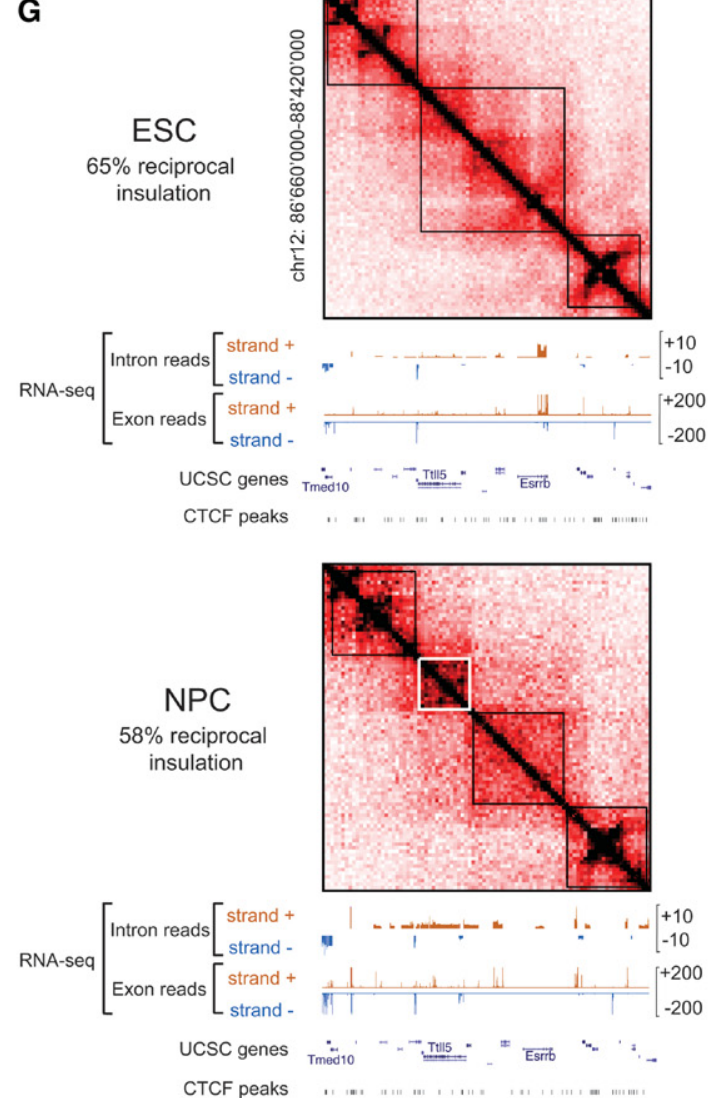

Figure 3. Transcriptional coregulation defines a functional privileged scale. $(A)$ Schematic representation of the definition of statistical enrichment in the number of coregulated domains. A domain is down- (up-) coregulated if its number of down- (up-) coregulated genes is larger than in $95 \%$ of cyclic permutated genomes (empirical $P \leq 0.05$ ). A $Z$-score is calculated as the difference between the number of coregulated domains detected in the real genome $\left(\mathrm{N}^{\text {obs }}\right)$ and the mean number of coregulated domains detected in 2000 randomized genomes $\left(\mathrm{N}^{\mathrm{exp}}\right)$, weighted by its standard deviation $\sigma^{\text {exp }}$. ( $B$ ) Distribution of average fold changes in expression level for domains at different RI values. For each RI value, the number of domains that are either upor down-regulated during differentiation (at the $P \leq 0.05$ level) is also shown in the upper part of the graph. Box: $25 \%-75 \%$ range (black line: median). (C) The statistical enrichment in the number of down-regulated domains is plotted as a function of the RI threshold. Transcriptional coregulation is significant at any level below $\sim 70 \%$ RI but maximal at $65 \%$. (D) Same as panel $C$ for up-regulated domains. (E) Same analysis as in panel $C$ when using domains based on Hi-C data in NPCs. ( $F$ ) Same as panel $E$ for up-regulated domains. ( $G$ ) Example of domains that were created de novo during differentiation and detected only in the set of NPC TADs (58\% RI).

were defined de novo during differentiation in parallel with a significant increase in their genomic activity (Fig. 3G; Supplemental Fig. S3d). This might suggest that TADs in NPCs are more predictive of transcriptional coregulation of up-regulated genes because domains that are transcriptionally active in the final state of differentiation can only be precisely detected when they are active (i.e., in the final state) but do not appear as defined in the set of TADs in the initial state. These domains represent extreme cases that 
illustrate that increased genomic activity can be associated with increased structural complexity, and in this case, with de novo formation of local structures. This is reminiscent of what was observed on the inactive $X$ chromosome (Giorgetti et al. 2016), where the presence of TAD-like structures is only observed in the context of gene activation.

\section{Enhancer-promoter communication is optimized at the scale of TADs}

The finding that TADs emerge as the folding scale that maximizes transcriptional coregulation but are not an intrinsically defined structural level (cf. Fig. 1) prompted us to ask whether TADs specifically favor enhancer-promoter communication in ESCs. We analyzed available ChIP-seq data sets (Supplemental Table S1) to identify enhancers based on H3K27ac, H3K4me1, and H3K4me3 patterns (see Supplemental Methods); active promoters were identified using the strand-specific total RNA-seq data sets generated in this study.

To check whether the presence of domains at each level in the hierarchy corresponds to gain (or loss) in interactions, we considered pairs of Hi-C bins containing enhancers and promoters. We then calculated the ratio between their Hi-C counts vs. the genomic average for loci separated by the same genomic distance (Fig. $4 \mathrm{~A})$. We observed substantial enrichment in interactions between enhancers and promoters within the same (active) domain up to $\sim 65 \%$ reciprocal insulation (Fig. $4 \mathrm{~B}$, red curve). Thus, TADs appear to be within the uppermost scales in the folding hierarchy where enhancer-promoter contacts are maximally enriched within domains. On the other hand, enhancer-promoter interactions are also enriched across boundaries with the two neighboring domains, until slightly below the scale of TADs (Fig. 4C, red curve). This reflects the fact that domains up to TADs are detected as increasingly bigger units, which are defined by the union of smaller subdomains found at lower insulation values where enhancer-promoter interactions are strongly enriched (cf. Fig. 4B). At higher re- ciprocal insulation, interactions across boundaries are depleted. CTCF-bond loci showed a similar pattern, with even higher levels of enrichment within domains and lower enrichment across domain boundaries (Fig. 4B,C, black curves). This result is obtained irrespective of the reciprocal orientation of pairs of CTCF motifs, although enrichments are globally higher for convergent CTCF sites (Supplemental Fig. S4a). Importantly, when we considered all pairs of loci within the same active domains where enhancers and promoters were identified, or random interactions drawn from the same distribution of distances as enhancer-promoter pairs, we observed a much lower increase in interactions inside domains (Fig. 4B, green and blue curves). Moreover, interactions across domains were also depleted at low insulation levels (Fig. $4 \mathrm{C})$. We obtained very similar results in NPCs and the CH12 cell line (Supplemental Fig. S4b,c).

Thus, TADs occur in the folding range where enhancer-promoter communication might be 'optimal,' i.e., enhancer-promoter contacts are maximally enriched within domains but begin to be depleted across domain boundaries.

\section{The local complexity in chromosomal folding correlates with transcriptional activity and CTCF binding}

We next used the CaTCH algorithm to quantify local chromosome folding complexity within each TAD and correlate it to the level of local transcriptional activity. To this aim, we first computed the number of hierarchical sublevels that can be identified within a domain (see Methods) as a measure for local folding complexity. We then used the RNA-seq profiles to assign transcripts to domains based on the genomic position of their promoters. We did not limit our analysis to the exonic signal (corresponding to mature mRNA), but we also considered the intronic reads, the latter being a more reliable measure of transcriptional activity (see Methods section). We found that at the level of single TADs, a quantitative correlation exists between the number of sublevels and both total (exonic) and unspliced mRNA reads per domain (Fig. 5A,B;
A

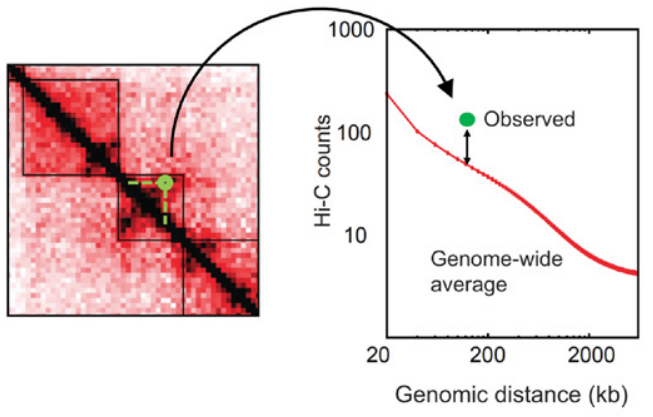

B

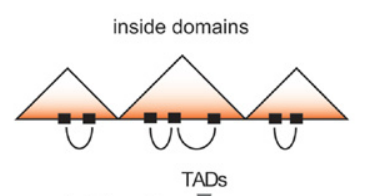

TADs

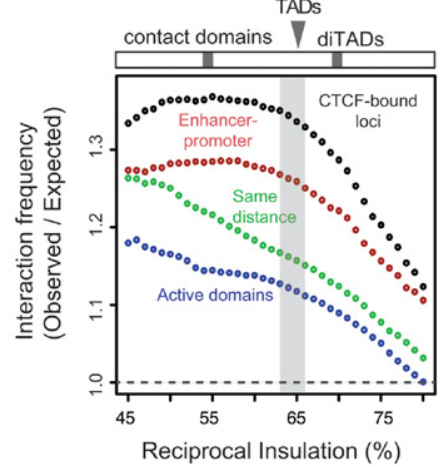

C

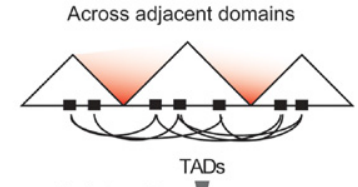
contact domains $\nabla$ diTADs

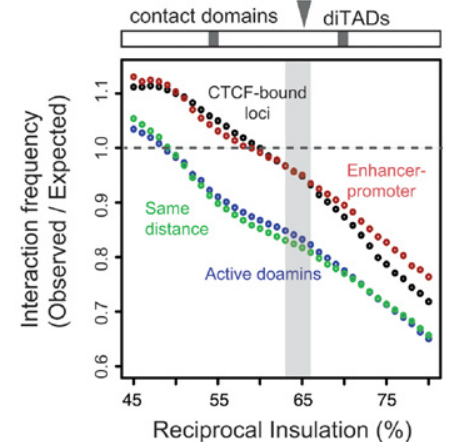

Figure 4. TADs define a scale where promoter-enhancer communication is optimal in ESCs. ( $A$ ) Schematics of contact enrichment analysis. For each pair of loci, we calculated the ratio between observed Hi-C counts and the genome-wide average counts for loci located at the same genomic distance. ( $B$ ) Enrichment in interactions between pairs of loci belonging to the same domain, as a function of reciprocal insulation. Colors refer to random loci within active TADs (blue), enhancer-promoter pairs (red), random loci with the same distance distribution as enhancer-promoter pairs (green), and CTCF-containing loci (black). Median enrichment over all pairs of considered loci are plotted. Gray shaded area indicates the $63 \%-66 \%$ confidence interval where maximal coregulation of genes occurs in partially reshuffled genomes (cf. Supplemental Fig. S3b). (C) Enrichment (or depletion) in interactions between pairs of loci, defined as in panel $A$ but located across consecutive domains. Gray shaded area as in panel $B$.

\section{Genome Research}

www.genome.org 
TADs are a functionally privileged folding scale

A

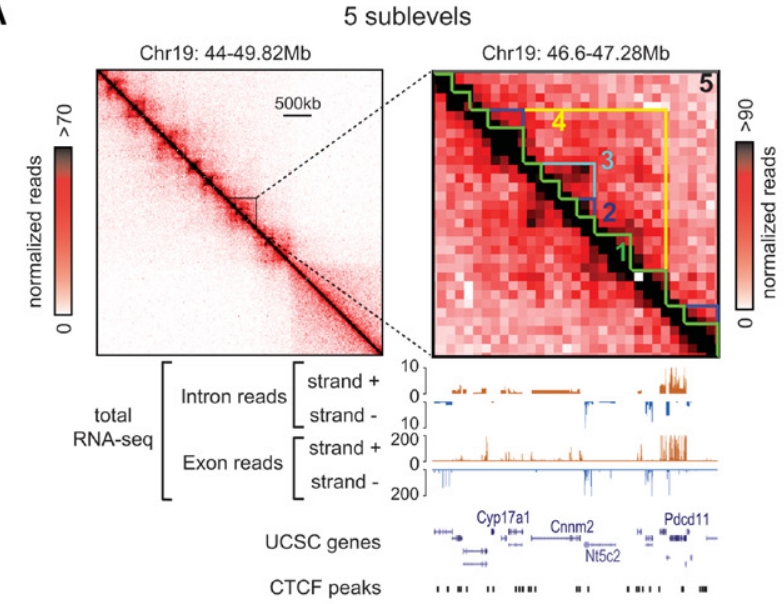

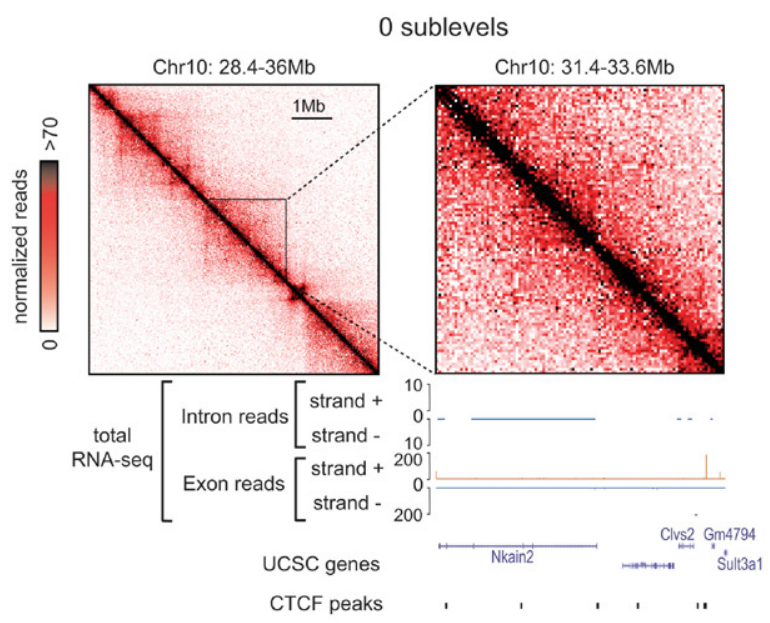

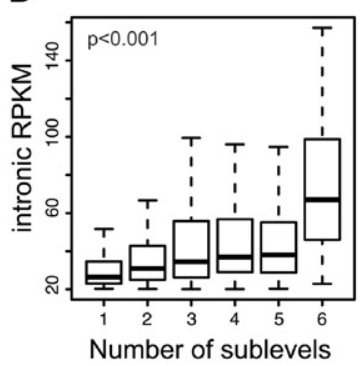

C

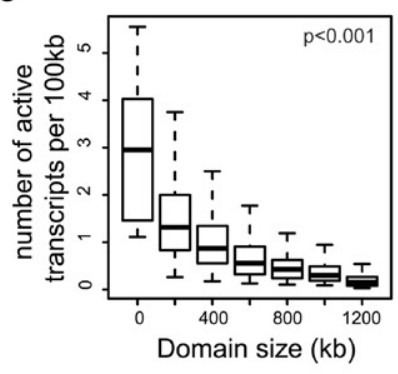

D

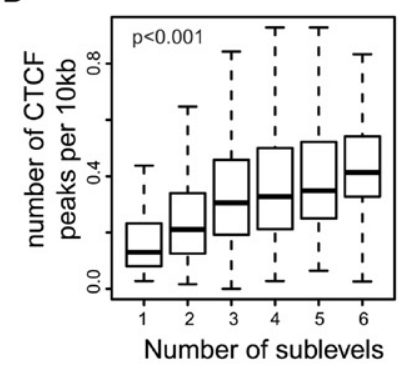

E

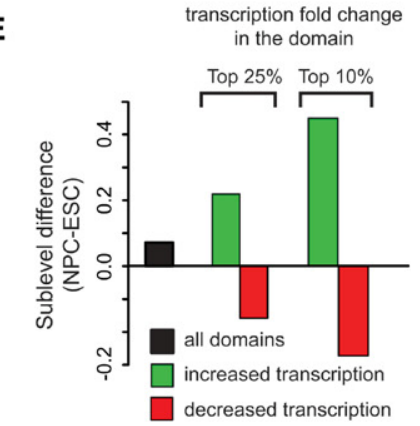

Figure 5. Local (changes in) folding complexity correlate with transcriptional activity in ESCs and during differentiation. ( $A$ ) Examples of regions with different levels of local folding complexity and correlated transcriptional activities. $(B)$ The number of sublevels in a domain correlates with the transcriptional activity within the domain (shown for domains at $65 \%$ RI in ESC). P-value: Student's $t$-test associated to Spearman's correlation coefficient. (C) Smaller domains tend to be denser in actively transcribed genes and therefore globally more active than larger domains (shown for domains at $65 \%$ $\mathrm{RI}$ ). (D) The number of sublevels in a domain correlates with the density of CTCF-bound sites within the domain (shown for domains at $65 \% \mathrm{RI}$ ). ( $E$ ) Local changes in transcriptional activities during differentiation from ESCs to NPCs correspond to changes in local hierarchical complexity (see Methods). Differences in the number of hierarchical sublevels are shown for the $25 \%$ and $10 \%$ most up- or down-regulated domains identified at $65 \%$ $\mathrm{RI}$ in ESC.

Supplemental Fig. S5a). The number of sublevels also correlates with the mean transcriptional level per gene (Supplemental Fig. $\mathrm{S} 5 \mathrm{~b})$ and with the number of transcribed promoters in the domain (Supplemental Fig. S5c). We also observed that smaller TADs tend to be denser in actively transcribed genes (Fig. 5C) and are globally more active than larger domains (Supplemental Fig. S5d). In addition, the number of sublevels correlates with the density of CTCF ChIP-seq peaks within the domain (Fig. 5D).

These observations would predict that, during differentiation from ESCs to NPCs, local changes in transcriptional activities should correspond at least in part to changes in local folding complexity. To verify this hypothesis, we considered the set of TADs defined in ESCs and studied the changes in the number of sublevels in the same regions in NPCs. We found indeed that domains where transcriptional activity increases during differentiation tend to increase their internal structural complexity and vice versa, as exemplified by the average change in the number of sublevels in the most dynamic TADs (Fig. 5E; Supplemental Fig. S5e).

Finally, given that the local transcriptional activity and CTCF occupancy modulate folding complexity within single domains, we reasoned that sharp transitions in these quantities across domain boundaries could also contribute to domain segregation. By definition, each domain level in the folding hierarchy (including TADs) is defined by the minimal reciprocal insulation of its con- stituent domains. Thereby, each TAD in ESCs is insulated from its neighbors by at least $65 \%$. Interestingly, we found that, at the level of single TADs, reciprocal insulation correlates with the difference in transcriptional activity and CTCF occupancy within vs. across its borders (Supplemental Fig. S5f,g). Similar results were found when considering all other levels in the hierarchy, either at lower or higher levels of insulation compared to TADs (Supplemental Fig. S5f). Thus, sharper transitions in the genomic density of CTCF binding sites and transcribed genes correspond to stronger boundaries between adjacent domains.

\section{Discussion}

Determining how enhancers exert their regulatory functions on distal promoters critically depends upon our level of understanding of the three-dimensional organization of chromatin. Several studies have provided evidence on the fundamental role of compartmentalization into TADs to instruct enhancer-promoter communication (Nora et al. 2012; Symmons et al. 2014; Lupiáñez et al. 2015; Franke et al. 2016), but they remain elusive on what makes TADs 'special' compared to other chromosomal folding layers, such as sub-TADs and notably contact domains or meta-TADs. In this study, we present a new domain-calling algorithm that is able to segment Hi-C interaction maps into nested sets of 
topologically associating domains, based on their reciprocal physical insulation. Our approach to partition the genome into nested sets of domains has two main advantages over existing hierarchical TAD callers (Filippova et al. 2014; Lévy-Leduc et al. 2014; Shin et al. 2015; Weinreb and Raphael 2015; Chen et al. 2016; Shavit et al. 2016): (1) The CaTCH algorithm does not rely on any free parameters, except reciprocal insulation itself that is used to stratify the domains; (2) Unlike other methods that identify hierarchies of domains, where parameters have an unclear structural or biological interpretation, reciprocal insulation estimates how well a domain is segregated from its neighbors. $\mathrm{CaTCH}$ is fast and requires less computing power: Identifying a whole hierarchy of domains on a single $100-\mathrm{Mb}$ chromosome takes $<4$ min on a single CPU, starting from mouse Hi-C data at $20-\mathrm{kb}$ resolution. We note that reciprocal insulation is conceptually similar to the 'local contrast' measure introduced in Van Bortle et al. (2014); here, however, we used the parameter to define a full hierarchical tree of domains, rather than employing it to characterize the strength of boundaries of a given set of domains.

By applying CaTCH to published Hi-C data sets, we were able to show that previously reported topological structures are detected by the algorithm as differentially insulated levels within a continuous hierarchy of nested folding layers (Fig. 1). This gave us the possibility to compare all levels simultaneously in terms of their structural and functional properties. Based on purely structural characteristics of the domains detected over the entire mouse genome, we found that none of these sets constitutes an intrinsically privileged scale. However, we observed that the scale of TADs emerges as a privileged functional one, where fundamental properties previously associated with TADs and notably related to their role in long-range transcriptional regulation are maximized.

CTCF clustering at domain boundaries has been repeatedly reported as one of the hallmarks of topological domains across species (Dixon et al. 2012; Sexton et al. 2012; Van Bortle et al. 2014; Vietri Rudan et al. 2015). In agreement with that, we show that maximal CTCF clustering at boundaries is highly predictive of the set of domains with the most conserved boundaries across cell types (Fig. 2). In fact, finding hierarchical levels with $~ 3400$ domains seems to provide a sufficient operational criterion to identify the TAD scale when using CaTCH (Fig. 2D), even in the absence of matched CTCF ChIP-seq data sets.

The resolution of our data set $(20 \mathrm{~kb})$ does not enable the detecting of looping interactions between single CTCF sites that can be found in very high-resolution Hi-C (Rao et al. 2014) or ChIAPET experiments (Tang et al. 2015), and it is therefore not possible to assess the precise reciprocal orientation of CTCF site clusters that occur within domain boundaries. However, between 15\% and $22 \%$ of the most internal CTCF site pairs at the boundaries of TADs are convergent, which represents a maximum across the entire folding hierarchy (Supplemental Fig. S2f).

Although boundary-associated CTCF might play an important role in defining domains and in particular TADs, CTCF also pervasively binds within domains. Within a given hierarchical level and TADs in particular, domains that are more reciprocally insulated tend to have a higher imbalance in the number of CTCFbound sites within vs. across their boundaries. Notably, regions that are highly bound by CTCF and are flanked by low-occupancy domains are highly insulated from the flanking regions (see, for example, Supplemental Fig. S5g, right). In addition, the density of CTCF-bound sites within a domain correlates with the hierarchical complexity of topological domains at all scales, including TADs (Fig. 5). Together with the fact that the hierarchical complexity also correlates with the overall transcriptional activity of a domain, this is in line with earlier findings that sub-TAD structures are strongly associated with CTCF-bound sites and active regulatory sequences (Phillips-Cremins et al. 2013). However, our results also suggest that interactions mediated by CTCF (and possibly additional factors associated with active regulatory sequences) within transcriptionally active domains play an important role in modulating the strength of boundaries between adjacent domains. Strong asymmetry in CTCF occupancy and transcriptional activity across boundaries can arise as a consequence of marked transitions in gene density and/or number of regulatory sequences. In addition, asymmetry can occur corresponding to cell-type-specific transitions in genomic activity between adjacent TADs (cf. Supplemental Fig. S5g, right panel). This in turn might be driven by transitions in the enrichment for cell-type-specific regulatory sequences (such as binding sites for lineage-determining transcription factors) across the boundary between the two TADs.

TADs appear in the uppermost layers in the folding hierarchy where interactions within active domains specifically, and between enhancers and promoters in particular, are strongly enriched compared to the genome-wide average interactions (Fig. 4). On the other hand, interactions across the boundaries of active TADs start to be depleted as compared to genome-wide averages. TADs thus appear to belong to the domain scale where a trade-off is established between maximizing interactions within the interior of domains and not enriching interactions across domain boundaries. In this light, it is remarkable that TADs emerge as the set of domains where the coregulation of genes during differentiation is maximal (Fig. 4). Although the precise mechanisms that govern enhancer action on promoters is still unknown, it is tempting to speculate that rather than absolute interaction frequency, the balance between interactions within and across domains determines the genomic range of action of enhancers, and this could contribute at least in part to establishing higher transcriptional coregulation at the level of TADs.

\section{Methods}

\section{Hi-C data sets}

ESCs and NPCs Hi-C data sets were obtained from Giorgetti et al. (2016). Reads from 129Sv and Cast/EiJ alleles were combined to increase coverage, and data were binned at 20-kb resolution. CH12 data are from Rao et al. (2014), binned at $10 \mathrm{~kb}$. Mouse fetal liver $\mathrm{Hi}-\mathrm{C}$ data are from Nagano et al. (2015), binned at $25 \mathrm{~kb}$. ESC, NPC, and liver Hi-C were normalized with iterative correction (Imakaev et al. 2012). CH12 data were normalized with the VCSQRT method (Rao et al. 2014).

\section{The $\mathrm{CaTCH}$ algorithm}

The algorithm takes a normalized Hi-C matrix as an input, binned at an arbitrary resolution $\mathrm{r}$. The genome is first partitioned into domain seeds of size $2^{*} \mathrm{r}$, which are progressively merged into larger domains. Reciprocal insulation (RI) is defined as in Eq. (1) in the main text. Given a threshold on RI, two consecutive domains are merged into one if their RI is smaller than the threshold. Increasing the RI threshold from $0 \%$ to $100 \%$ in steps of $0.1 \%$ results in increasingly larger domains. To lose memory of the initial partitioning of the genome into domain seeds, small shifts (two genomic bins) in domain boundaries are allowed at each step. Finally, to avoid that the discrete increase in RI threshold $(0.1 \%$ steps) results in a final domain tree that depends on the order of mergings and is therefore not unique, we impose a rule on merging

\section{Genome Research}

www.genome.org 
order: A domain can be merged with either the one that precedes or the one that follows it along the genome; the pair with lowest RI is merged first (see Supplemental Methods).

\section{Computationally generated contact maps with preferential folding levels}

Control contact maps with one or two folding levels were created by generating a power law decreasing contact map for each level, to which Gaussian random noise is added (see Supplemental Methods). The contact map with zero folding layers was generated by replacing the actual Hi-C counts in the contact map for Chr 19 in ESCs with the average genome-wide counts for loci with the same genomic distance and adding Gaussian noise.

\section{Cell culture}

Culture of the female mouse ES cell line F121.6 (129Sv-Cast/EiJ) and NPC clone analyzed in Giorgetti et al. (2016) was performed as previously described (Gendrel et al. 2014; Giorgetti et al. 2016). All cell lines used in this study were characterized for absence of mycoplasma contamination.

\section{RNA-seq data and analysis and other analyses of genomic data}

Strand-specific total RNA-seq libraries from two biological replicates of ESCs and NPCs were prepared with the ScriptSeq v2 kit (Illumina) and sequenced on an Illumina HiSeq 2000 for a total of $\sim 30$ million uniquely aligned reads per sample. Samples were aligned to mouse mm9. For details on the RNA-seq and ChIP-seq analysis, CTCF motif assignment, and enhancer calling, please refer to Supplemental Methods.

\section{Definition of hierarchical sublevels}

A subregion within a domain at any scale in the folding hierarchy was defined as a sublevel if it is detected as a domain over more than $>5 \%$ of the preceding reciprocal insulation thresholds. $P$-values in transcription and CTCF content vs. number of sublevels (Fig. 5) were obtained using the function cor.test in R (Spearman method) and represent the results of Student's $t$-tests on the Spearman's correlation coefficient.

\section{Analysis of structural reorganization during differentiation}

We focused on TADs defined in ESCs and defined the number of sublevels detected in NPCs in the corresponding regions, using NPC domains below 58\% since those are the domains that best match domains at $65 \%$ in ESCs (see Supplemental Fig. S2g). We estimated the local amount of structural reorganization as the change in the number of sublevels between ESCs and NPCs.

\section{Analysis of enhancer-promoter interactions}

Genomic 20-kb (ESCs and NPCs) and 10-kb (CH12) bins were assigned to 'enhancer,' 'promoter,' or 'CTCF' categories if they contain at least one of these elements (see Supplemental Methods). If a bin shows multiple classifications, it was assigned to all categories. In the analysis for Figure 4, in order to avoid including undersampled interactions due to limited Hi-C coverage at large genomic distances, we only considered pairs of loci separated by $<2$ $\mathrm{Mb}$ in ESCs and NPCs, and $1 \mathrm{Mb}$ in CH12 cells. Cutoffs were chosen to exclude genomic distances where average Hi-C counts are dominated by experimental noise (Supplemental Fig. S4d).

\section{Data access}

The sequencing data from this study have been submitted to the NCBI Gene Expression Omnibus (GEO; http://www.ncbi.nlm. nih.gov/geo/) under accession number GSE84724. CaTCH is provided as an $\mathrm{R}$ package at https://github.com/zhanyinx/ CaTCH_R. Source code can be found in Supplemental Methods.

\section{Acknowledgments}

Research in the Giorgetti lab was supported by the Novartis Research Foundation. Initial analyses for this study were conceived and established in Edith Heard's laboratory, Institut Curie and PSL (Paris), where L.M.'s salary was paid for by an EMBO ASTF 5632012 fellowship to L.M. and SyBoSS 62012 grant to Edith Heard. We thank Federico Comoglio for assistance on code development and for critically reading the manuscript, Stéphane Thiry and Tim Roloff for assistance on RNA-seq library preparation and sequencing, Edith Heard for critically reading the manuscript, NIBR computing resources and Stefan Grzybek for help with cluster and server supports, and Mikael Attia for cell culture. We acknowledge The ENCODE Project Consortium and in particular the Ren and Hardison laboratories for ChIP-Seq data sets in ESC and CH12, and the Myers laboratory for ChIP-seq data sets in fetal liver cells.

Author contributions: Y.Z. wrote the code and performed all analyses with assistance from G.T., L.M., and M.S.; L.M., E.G.S., and N.B. set up the preliminary analyses and discussed the results; I.B. performed enhancer calling and discussed the results; G.T. assisted with data analysis. L.G. prepared RNA samples. L.G. designed the study. L.G. and Y.Z. wrote the paper.

\section{References}

Belton J-M, McCord RP, Gibcus JH, Naumova N, Zhan Y, Dekker J. 2012. Hi$\mathrm{C}$ : a comprehensive technique to capture the conformation of genomes. Methods 58: 268-276.

Berlivet S, Paquette D, Dumouchel A, Langlais D, Dostie J, Kmita M. 2013. Clustering of tissue-specific sub-TADs accompanies the regulation of HoxA genes in developing limbs. PLoS Genet 9: e1004018.

Chen J, Hero AO, Rajapakse I. 2016. Spectral identification of topological domains. Bioinformatics 32: 2151-2158.

Cheng Y, Ma Z, Kim B-H, Wu W, Cayting P, Boyle AP, Sundaram V, Xing X, Dogan N, Li J, et al. 2014. Principles of regulatory information conservation between mouse and human. Nature 515: 371-375.

de Wit E, Vos ESM, Holwerda SJB, Valdes-Quezada C, Verstegen MJAM, Teunissen H, Splinter E, Wijchers PJ, Krijger PHL, de Laat W. 2015. CTCF binding polarity determines chromatin looping. Mol Cell 60: $676-684$.

Dixon JR, Selvaraj S, Yue F, Kim A, Li Y, Shen Y, Hu M, Liu JS, Ren B. 2012. Topological domains in mammalian genomes identified by analysis of chromatin interactions. Nature 485: 376-380.

Filippova D, Patro R, Duggal G, Kingsford C. 2014. Identification of alternative topological domains in chromatin. Algorithms Mol Biol 9: 14

Franke M, Ibrahim DM, Andrey G, Schwarzer W, Heinrich V, Schöpflin R, Kraft K, Kempfer R, Jerković I, Chan W-L, et al. 2016. Formation of new chromatin domains determines pathogenicity of genomic duplications. Nature 538: 265-269.

Fraser J, Ferrai C, Chiariello AM, Schueler M, Rito T, Laudanno G, Barbieri M, Moore BL, Kraemer DC, Aitken S, et al. 2015. Hierarchical folding and reorganization of chromosomes are linked to transcriptional changes in cellular differentiation. Mol Syst Biol 11: 852 .

Fudenberg G, Mirny LA. 2012. Higher-order chromatin structure: bridging physics and biology. Curr Opin Genet Dev 22: 115-124.

Gendrel A-V, Attia M, Chen C-J, Diabangouaya P, Servant N, Barillot E, Heard E. 2014. Developmental dynamics and disease potential of random monoallelic gene expression. Dev Cell 28: 366-380.

Gibcus JH, Dekker J. 2013. The hierarchy of the 3D genome. Mol Cell 49: 773-782.

Giorgetti L, Galupa R, Nora EP, Piolot T, Lam F, Dekker J, Tiana G, Heard E. 2014. Predictive polymer modeling reveals coupled fluctuations in chromosome conformation and transcription. Cell 157: 950-963. 
Giorgetti L, Lajoie BR, Carter AC, Attia M, Zhan Y, Xu J, Chen CJ, Kaplan N, Chang HY, Heard E, et al. 2016. Structural organization of the inactive X chromosome in the mouse. Nature 535: 575-579.

Guo Y, Xu Q, Canzio D, Shou J, Li J, Gorkin DU, Jung I, Wu H, Zhai Y, Tang $\mathrm{Y}$, et al. 2015. CRISPR inversion of CTCF sites alters genome topology and enhancer/promoter function. Cell 162: 900-910.

Hastie T, Tibshirani R, Friedman J. 2009. The elements of statistical learning Springer, New York. http://link.springer.com/10.1007/978-0-38784858-7.

Hou C, Li L, Qin ZS, Corces VG. 2012. Gene density, transcription, and insulators contribute to the partition of the Drosophila genome into physical domains. Mol Cell 48: 471-484.

Imakaev M, Fudenberg G, McCord RP, Naumova N, Goloborodko A, Lajoie BR, Dekker J, Mirny LA. 2012. Iterative correction of Hi-C data reveals hallmarks of chromosome organization. Nat Methods 9: 999-1003.

Junier I, Spill YG, Marti-Renom MA, Beato M, le Dily F. 2015. On the demultiplexing of chromosome capture conformation data. FEBS Lett 589: 3005-3013.

Le Dily F, Baù D, Pohl A, Vicent GP, Serra F, Soronellas D, Castellano G, Wright RHG, Ballare C, Filion G, et al. 2014. Distinct structural transitions of chromatin topological domains correlate with coordinated hormone-induced gene regulation. Genes Dev 28: 2151-2162.

Lévy-Leduc C, Delattre M, Mary-Huard T, Robin S. 2014. Two-dimensiona segmentation for analyzing Hi-C data. Bioinformatics 30: i386-i392.

Lieberman-Aiden E, van Berkum NL, Williams L, Imakaev M, Ragoczy T, Telling A, Amit I, Lajoie BR, Sabo PJ, Dorschner MO, et al. 2009. Comprehensive mapping of long-range interactions reveals folding principles of the human genome. Science 326: 289-293.

Lupiáñez DG, Kraft K, Heinrich V, Krawitz P, Brancati F, Klopocki E, Horn D Kayserili H, Opitz JM, Laxova R, et al. 2015. Disruptions of topological chromatin domains cause pathogenic rewiring of gene-enhancer interactions. Cell 161: 1012-1025.

Merkenschlager M, Nora EP. 2016. CTCF and cohesin in genome folding and transcriptional gene regulation. Annu Rev Genomics Hum Genet 17: $17-43$.

Nagano T, Lubling Y, Stevens TJ, Schoenfelder S, Yaffe E, Dean W, Laue ED, Tanay A, Fraser P. 2013. Single-cell Hi-C reveals cell-to-cell variability in chromosome structure. Nature 502: 59-64.

Nagano T, Várnai C, Schoenfelder S, Javierre B-M, Wingett SW, Fraser P. 2015. Comparison of Hi-C results using in-solution versus in-nucleus ligation. Genome Biol 16: 175.

Nora EP, Lajoie BR, Schulz EG, Giorgetti L, Okamoto I, Servant N, Piolot T, van Berkum NL, Meisig J, Sedat J, et al. 2012. Spatial partitioning of the regulatory landscape of the X-inactivation centre. Nature 485: 381-385.

Phillips-Cremins JE, Sauria MEG, Sanyal A, Gerasimova TI, Lajoie BR, Bell JSK, Ong C-T, Hookway TA, Guo C, Sun Y, et al. 2013. Architectural pro- tein subclasses shape 3D organization of genomes during lineage commitment. Cell 153: 1281-1295.

Rao SSP, Huntley MH, Durand NC, Stamenova EK, Bochkov ID, Robinson JT, Sanborn AL, Machol I, Omer AD, Lander ES, et al. 2014. A 3D map of the human genome at kilobase resolution reveals principles of chromatin looping. Cell 159: 1665-1680.

Sanborn AL, Rao SSP, Huang S-C, Durand NC, Huntley MH, Jewett AI, Bochkov ID, Chinnappan D, Cutkosky A, Li J, et al. 2015. Chromatin extrusion explains key features of loop and domain formation in wild-type and engineered genomes. Proc Natl Acad Sci 112: E6456-E6465.

Sexton T, Yaffe E, Kenigsberg E, Bantignies F, Leblanc B, Hoichman M, Parrinello H, Tanay A, Cavalli G. 2012. Three-dimensional folding and functional organization principles of the Drosophila genome. Cell 148: $458-472$.

Shavit Y, Walker BJ, Lio' P. 2016. Hierarchical block matrices as efficient representations of chromosome topologies and their application for 3C data integration. Bioinformatics 32: 1121-1129.

Shin H, Shi Y, Dai C, Tjong H, Gong K, Alber F, Zhou XJ. 2015. TopDom: an efficient and deterministic method for identifying topological domains in genomes. Nucleic Acids Res 44: e70.

Symmons O, Uslu VV, Tsujimura T, Ruf S, Nassari S, Schwarzer W, Ettwiller L, Spitz F. 2014. Functional and topological characteristics of mammalian regulatory domains. Genome Res 24: 390-400.

Tang Z, Luo OJ, Li X, Zheng M, Zhu JJ, Szalaj P, Trzaskoma P, Magalska A, Wlodarczyk J, Ruszczycki B, et al. 2015. CTCF-mediated human 3D genome architecture reveals chromatin topology for transcription. Cell 163: $1611-1627$.

Ulianov SV, Khrameeva EE, Gavrilov AA, Flyamer IM, Kos P, Mikhaleva EA Penin AA, Logacheva MD, Imakaev MV, Chertovich A, et al. 2015. Active chromatin and transcription play a key role in chromosome partitioning into topologically associating domains. Genome Res 26: 70-84

Van Bortle K, Nichols MH, Li L, Ong C-T, Takenaka N, Qin ZS, Corces VG. 2014. Insulator function and topological domain border strength scale with architectural protein occupancy. Genome Biol 15: R82.

Vietri Rudan M, Barrington C, Henderson S, Ernst C, Odom DT, Tanay A, Hadjur S. 2015. Comparative Hi-C reveals that CTCF underlies evolution of chromosomal domain architecture. Cell Rep 10: 1297-1309.

Weinreb C, Raphael BJ. 2015. Identification of hierarchical chromatin domains. Bioinformatics 32: 1601-1609.

Received July 15, 2016; accepted in revised form January 4, 2017. 


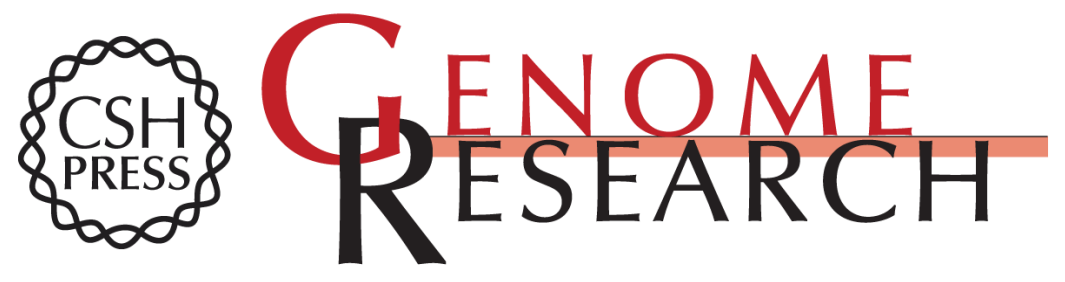

\section{Reciprocal insulation analysis of $\mathrm{Hi}-\mathrm{C}$ data shows that TADs represent a functionally but not structurally privileged scale in the hierarchical folding of chromosomes}

Yinxiu Zhan, Luca Mariani, Iros Barozzi, et al.

Genome Res. 2017 27: 479-490 originally published online January 5, 2017

Access the most recent version at doi:10.1101/gr.212803.116

Supplemental
Material http://genome.cshlp.org/content/suppl/2017/02/21/gr.212803.116.DC1

References This article cites 38 articles, 5 of which can be accessed free at: http://genome.cshlp.org/content/27/3/479.full.html\#ref-list-1

Creative This article is distributed exclusively by Cold Spring Harbor Laboratory Press for the Commons License

Email Alerting Service first six months after the full-issue publication date (see

http://genome.cshlp.org/site/misc/terms.xhtml). After six months, it is available under a Creative Commons License (Attribution-NonCommercial 4.0 International), as described at http://creativecommons.org/licenses/by-nc/4.0/.

Receive free email alerts when new articles cite this article - sign up in the box at the top right corner of the article or click here.

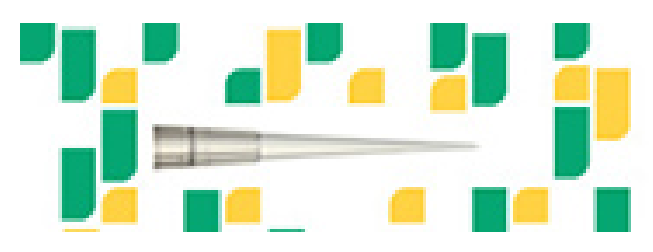

Focused on your science.

To subscribe to Genome Research go to:

https://genome.cshlp.org/subscriptions 